ARTICLE

DOI: $10.1038 / s 41467-017-00816-w$

\title{
Ultrafast giant magnetic cooling effect in ferromagnetic Co/Pt multilayers
}

Je-Ho Shim,2,3, Akbar Ali Syed ${ }^{2,3}$, Chul-Hoon Kim²,3,4, Kyung Min Lee ${ }^{5}$, Seung-Young Park ${ }^{6}$, Jong-Ryul Jeong ${ }^{5}$, Dong-Hyun $\mathrm{Kim}^{1}$ \& Dong Eon Kim²,3

The magnetic cooling effect originates from a large change in entropy by the forced magnetization alignment, which has long been considered to be utilized as an alternative environment-friendly cooling technology compared to conventional refrigeration. However, an ultimate timescale of the magnetic cooling effect has never been studied yet. Here, we report that a giant magnetic cooling (up to $200 \mathrm{~K}$ ) phenomenon exists in the Co/Pt nanomultilayers on a femtosecond timescale during the photoinduced demagnetization and remagnetization, where the disordered spins are more rapidly aligned, and thus magnetically cooled, by the external magnetic field via the lattice-spin interaction in the multilayer system. These findings were obtained by the extensive analysis of time-resolved magneto-optical responses with systematic variation of laser fluence as well as external field strength and direction. Ultrafast giant magnetic cooling observed in the present study can enable a new avenue to the realization of ultrafast magnetic devices.

\footnotetext{
${ }^{1}$ Department of Physics, Chungbuk National University, Cheongju 361-763, South Korea. ${ }^{2}$ Department of Physics and Center for Attosecond Science and Technology, POSTECH, Pohang 790-784, South Korea. ${ }^{3}$ Max Planck Center for Attosecond Science, Max Planck POSTECH/KOREA Research Initiative, Pohang 790-784, South Korea. ${ }^{4}$ Department of Advanced Materials Chemistry, Korea University, Sejong 339-700, South Korea. ${ }^{5}$ Department of Material Science and Engineering and Graduate School of Energy Science and Technology, Chungnam National University, Daejeon 305-764, South Korea. ${ }^{6}$ Spin Engineering Physics Team, Korea Basic Science Institute, Daejeon 305-806, South Korea. Correspondence and requests for materials should be addressed to D.-H.K. (email: donghyun@cbnu.ac.kr) or to D.E.K. (email: kimd@postech.ac.kr)
} 
S ince the discovery of the giant magnetocaloric effect ${ }^{1,2}$, there has been considerable interest in understanding the fundamental magnetic cooling mechanism ${ }^{3-8}$. The core process of the magnetic cooling effect (MCE) is that a material temperature is reduced as a result of the forced alignment of the magnetic moments by an external magnetic field. So far, most studies on the MCE have been carried out on bulk oxide materials with specific structures, such as perovskite or Heusler type, because the maximal MCE is normally observed around the structural and/or magnetic phase transitions ${ }^{3-6}$. Recently, MCE has also been observed for nanostructured films, such as $\mathrm{La}_{0.7} \mathrm{Ca}_{0.3} \mathrm{MnO}_{3}$ film ${ }^{7}$ and vanadium oxide superlattice films on graphene ${ }^{8}$, where the underlying mechanism for the observed MCE results is still based on a structural phase transition.

Because the MCE is the result of forced magnetization alignment by an external field, the question of how fast the MCE can be realized naturally arises. The answer to the question must involve the dynamics of demagnetization and subsequent remagnetization of magnetic materials. Over the past two decades, an ultrafast photoinduced demagnetization-remagnetization phenomenon via the time-resolved magneto-optical Kerr effect (TR-MOKE) has been investigated intensively since the seminal work by Beaurepaire et al. ${ }^{9}$. The ultrafast demagnetization and remagnetization process of the net magnetic moments triggered by light pulses has inspired substantial interest in the ultrafast interaction dynamics among spin, electron, lattice and photon on a femtosecond timescale. Quite recently, femtoseond laser heating was reported to play an important role in determining the ultrafast photoinduced demagnetization and remagnetization phenomenon for ferrimagnetic $\mathrm{GdFeCo}^{10,11}$ and ferromagnetic $\mathrm{FePt}^{12}$. Therefore, confirmation of the existence of the MCE during femtosecond laser heating becomes essential for further understanding the relevant spin, electron and lattice dynamics. On the other hand, no work has been done to answer the question as to how fast MCE can be realized if the MCE exists during the photoinduced demagnetization and remagnetization process. To reveal the existence of MCE on the ultrafast timescale, it is important to track the spin, electron and lattice temperatures separately.

In the case of ferromagnetic metallic films, the characteristic demagnetization time is known phenomenologically to be shorter than the electron relaxation time $\mathrm{e}^{13,14}$ for most systems, so that the spin temperature is assumed to instantly follow the electron temperature ${ }^{14-17}$. However, the assumption of the same temperatures for spin and electron in ferromagnetic metallic film systems needs to be clarified since it has been reported that there is a substantial temperature difference between spin and electron for a half-metallic system ${ }^{18}$.

In the following, we report our experimental demonstration of the existence of the MCE phenomenon on a femtosecond timescale. TR-MOKE experiment has been carried out for ferromagnetic $\mathrm{Co} / \mathrm{Pt}$ multilayers, where the 3 -temperature model $(3 \mathrm{TM})^{9}$ was applied to separately track the temperatures of spin, electron and lattice after being excited by femtosecond laser. From our systematic investigation with variation of external magnetic field strengths as well as pump fluences, it is concluded that the separated temperatures of spin, electron, and lattice and independent cooling of spin sub-system by the external magnetic field on a sub-picosecond timescale is considered to be a main origin of the ultrafast giant MCE. Ultrafast giant MCE by the forced alignment of magnetization under external magnetic fields is found to be a universal phenomena, observed for all $\mathrm{Co} / \mathrm{Pt}$ multilayers with repeat number of 5, 10 and 15 . The existence of the giant MCE was also confirmed by exponential fitting of the relaxation behavior during the remagnetization of the $\mathrm{Co} / \mathrm{Pt}$ multilayer systems. a

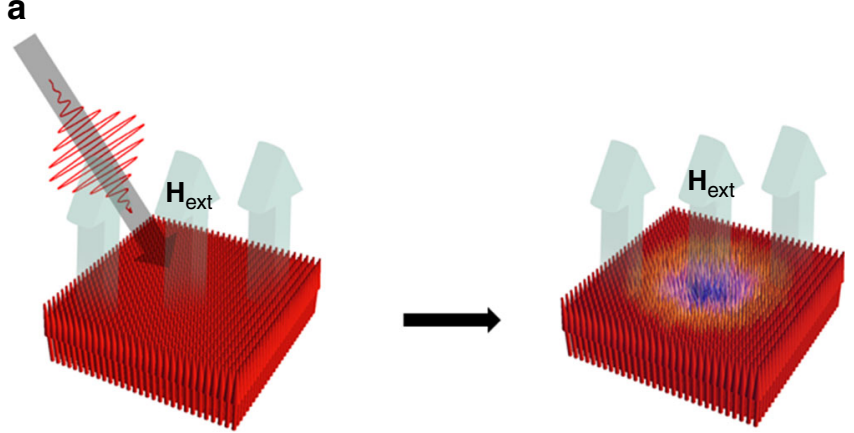

b

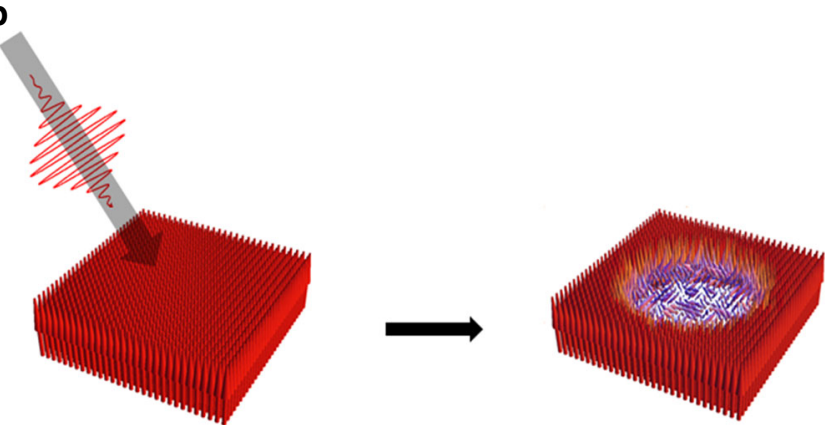

Fig. 1 Schematic diagram of the ultrafast magnetic cooling effect. a Upwardly saturated initial spin moments under an external magnetic field $\left(\boldsymbol{H}_{\text {ext }}\right)$ before a laser pulse excites the sample (left). Laser pulse propagation direction is represented by gray arrow. Demagnetized state after a laser pulse hits the sample under $\mathbf{H}_{\text {ext }}$ (right). b Upwardly saturated initial spin moments before a laser pulse excites the sample (left).

Demagnetized state after a laser pulse hits the sample under zero external magnetic field (right)

\section{Results}

TR-MOKE measurement. Figure 1 illustrates how a spin state demagnetized by a femtosecond laser pulse might be forced to be aligned under an external magnetic field, leading to a decrease in the effective spin temperature (Fig. 1a) compared to the case of demagnetization under a zero external field (Fig. 1b). To carry out a systematic investigation of this magnetic cooling phenomenon on a femtosecond time scale, it is important to have material parameters controlled in a desired manner. A ferromagnetic metallic multilayer provides an interesting playground to explore the above mechanism because important magnetic properties, such as the perpendicular magnetic anisotropy (PMA) and saturation magnetization, are engineered easily by controlling the multilayer parameters, such as the repeat number and sublayer thickness ${ }^{19-23}$. Another important feature of the ferromagnetic metallic multilayer is that there is no giant MCE originating from the oxide-related structural change. Therefore, if the MCE is measured in the ferromagnetic metallic multilayers, it must have purely originated from the forced alignment of magnetic moments by an external field.

We have measured TR-MOKE signal both with modulating pump-beam and with modulating probe-beam by a mechanical chopper during the stroboscopic measurement, where the systematic comparison is fully discussed in the Supplementary Notes 2-5. While the TR-MOKE signal measured with probebeam modulation simply detects a signal proportional to a magnetization with an improved signal-to-noise ratio $9,24,25$, the TR-MOKE signal measured with pump-beam modulation selectively detects a signal proportional to the change in the magnetization induced by the pump-beam rather than the magnetization itself $9,26-31$. It should be mentioned that the 

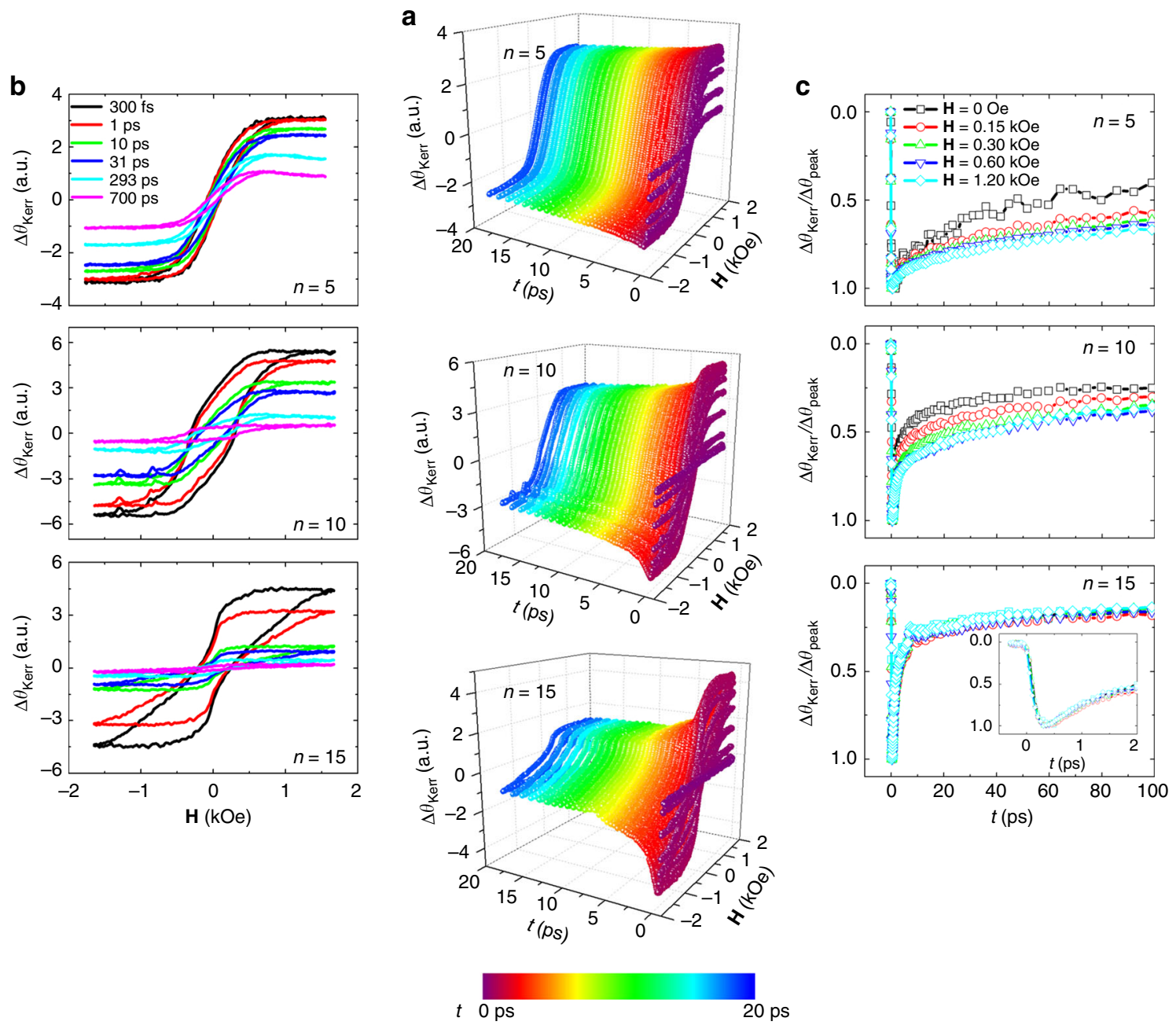
Fig. 2 Time-resolved magneto-optical Kerr effect signals. a 3D-map of TR-MOKE of [Co/Pt $]_{\mathrm{n}}$ with $n=5,10$, and 15 . The color code on the bottom indicates
a stroboscopically elapsed time from 0 to $40 \mathrm{ps}$. b Time-dependent $\Delta \theta_{\mathrm{Kerr}}$ hysteresis loops measured by pump-beam modulation at different delays from 300 fs to 700 ps for $n=5,10$ and 15. (c) Field-dependent normalized TR-MOKE signal ( $\left.\Delta \theta_{\text {Kerr }} / \Delta \theta_{\text {peak }}\right)$ for $n=5,10$ and 15 . The inset in the bottom shows a TR-MOKE signal within $2 \mathrm{ps}$

sum of the pump- and probe-beam modulated loops is to be invariant over all negative and positive time delays and confirmed experiments (Supplementary Note 4). Since the summed loop is constant, TR-MOKE signal either with pump- or with probebeam modulation can be analyzed without loss of generality. Here, we focus on the evolution of hysteresis loops measured with pump-beam modulation during the photoinduced demagnetization-remagnetization process.

In Fig. 2, the TR-MOKE signals during the demagnetization and remagnetization are also plotted with respect to the field $(\mathbf{H})$ and time $(t)$. $\mathbf{H}$ was applied with an angle of $23^{\circ}$ from the film normal. The MOKE signal vs. $\mathbf{H}$ (Fig. $2 \mathrm{~b}$ ) provides the timeresolved magnetic hysteresis loop at a specific delay and the temporal change in the MOKE signal (Fig. 2c) provides information at a specific applied field. The color code of Fig. $2 \mathrm{a}$ (purple to blue) represents the delay time between the time zero and $20 \mathrm{ps}$. Note also that the TR-MOKE feature becomes different for different repeat numbers. Generally, hysteresis loops represented by $\Delta \theta_{\text {Kerr }}$ (Fig. 2b) exhibit a rapid increase in the loop height until $t \sim 300 \mathrm{fs}$ and a slow decrease thereafter for all the films. On the other hand, the detailed relaxation behavior for $t>300 \mathrm{fs}$ is substantially different for different repeat numbers. The TR-MOKE signals shown in Fig. 2c exhibit the typical behavior of the rapid demagnetization around $300 \mathrm{fs}$, which is followed by slow relaxation on a long time scale lasting for up to few hundreds of picoseconds, leading to remagnetization characteristic of the type-I ferromagnetic system ${ }^{14}$. The inset in the bottom figure of Fig. 2c shows a TR-MOKE signal at various fields during the initial $2 \mathrm{ps}$, where the demagnetization reaches its peak $\sim t=300 \mathrm{fs}$ within the error range.

Interestingly, the photoinduced demagnetization-remagnetization phenomena also become different for different repeat numbers. For the multilayer film with $n=15$, a recovery of more than $50 \%$ was observed after just several picoseconds, while a slower recovery was observed as $\mathrm{n}$ decreases. Attention should be paid to the fact that the photoinduced demagnetization-remagnetization process becomes quite fielddependent in the case of $n=5$ and 10, whereas the process becomes less dependent on the external magnetic field strength in the case of $n=15$.

3TM analysis. To understand the observed features, the TR-MOKE hysteresis loops were analyzed in full details. Considering that the present results were obtained with the pump-beam modulation, TR-MOKE signals measured in the present setup are not proportional to the absolute magnetization value but to the change in the magnetization induced by the 
Table 1 List of fitting parameters with variation of pump fluences and external field angles

\begin{tabular}{|c|c|c|c|c|}
\hline & $9.9 \mathrm{~mJ} \mathrm{~cm}^{-2}\left(\theta_{\mathrm{H}}=0^{\circ}\right)$ & $13.2 \mathrm{~mJ} \mathrm{~cm}^{-2}\left(\theta_{\mathrm{H}}=0^{\circ}\right)$ & $16.5 \mathrm{~mJ} \mathrm{~cm}^{-2}\left(\theta_{\mathrm{H}}=0^{\circ}\right)$ & $13.2 \mathrm{~mJ} \mathrm{~cm}^{-2}\left(\theta_{\mathrm{H}}=23^{\circ}\right)$ \\
\hline$G_{e l}\left(10^{17} W^{-3} K^{-1}\right)$ & 2.00 & 1.00 & 1.00 & 0.40 \\
\hline$G_{e s}\left(10^{17} W^{-3} K^{-1}\right)$ & 30.0 & 10.0 & 5.50 & 10.0 \\
\hline $\mathrm{G}_{\mathrm{Is}}\left(10^{17} \mathrm{~W} \mathrm{~m}^{-3} \mathrm{~K}^{-1}\right)[\mathbf{H}=1.70 \mathrm{kOe}]$ & 11.4 & 3.00 & 1.32 & 2.20 \\
\hline \multicolumn{5}{|l|}{$[\mathrm{Co} / \mathrm{Pt}]_{15}$ multilayer } \\
\hline $\mathrm{G}_{\mathrm{Is}}\left(10^{17} \mathrm{~W} \mathrm{~m}^{-3} \mathrm{~K}^{-1}\right)[\mathbf{H}=0 \mathrm{Oe}]$ & 0.60 & 0.30 & 0.25 & 2.70 \\
\hline$G_{\mathrm{Is}}\left(10^{17} \mathrm{~W} \mathrm{~m}^{-3} \mathrm{~K}^{-1}\right)[\mathbf{H}=1.70 \mathrm{kOe}]$ & 1.60 & 0.90 & 0.75 & 3.30 \\
\hline
\end{tabular}

pump laser pulse. Therefore, the hysteresis loops in Fig. $2 \mathrm{~b}$ are not conventional magnetic hysteresis loops, providing the macroscopic parameters, such as remanent magnetization $\left(M_{\mathrm{R}}\right)$, saturation magnetization $\left(M_{\mathrm{S}}\right)$. Instead, they indicate how much the corresponding physical quantities are changed; i.e., $\Delta M_{\mathrm{R}}$ and $\Delta M_{\mathrm{S}}$, respectively.

For the measured TR-MOKE hysteresis loops, under $3 \mathrm{TM}^{9}$, the following equations were used to fit the experimental data:

$$
\begin{array}{r}
C_{\mathrm{e}}\left(T_{\mathrm{e}}\right) \frac{\mathrm{d} T_{\mathrm{e}}}{\mathrm{d} t}=-G_{\mathrm{el}}\left(T_{\mathrm{e}}-T_{\mathrm{l}}\right)-G_{\mathrm{es}}\left(T_{\mathrm{e}}-T_{\mathrm{s}}\right)+P(t), \\
C_{\mathrm{l}}\left(T_{\mathrm{l}}\right) \frac{\mathrm{d} T_{\mathrm{l}}}{\mathrm{d} t}=-G_{\mathrm{el}}\left(T_{\mathrm{l}}-T_{\mathrm{e}}\right)-G_{\mathrm{ls}}\left(T_{1}-T_{\mathrm{s}}\right)-K_{\mathrm{l}}\left(T_{\mathrm{l}}-300\right)^{3}, \\
C_{\mathrm{s}}\left(T_{\mathrm{s}}\right) \frac{\mathrm{d} T_{\mathrm{s}}}{\mathrm{d} t}=-G_{\mathrm{es}}\left(T_{\mathrm{s}}-T_{\mathrm{e}}\right)-G_{\mathrm{ls}}\left(T_{\mathrm{s}}-T_{\mathrm{l}}\right),
\end{array}
$$

where $T_{\mathrm{e}}, T_{\mathrm{s}}$ and $T_{1}$ are the electron, spin and lattice temperatures, respectively. $C_{\mathrm{e}}, C_{\mathrm{s}}$ and $C_{\mathrm{l}}$ are the specific heats of the electron, spin and lattice, respectively. $G_{\mathrm{el}}$ is the electron-lattice interaction parameter. $G_{\mathrm{es}}$ and $G_{\mathrm{ls}}$ are the electron-spin and lattice-spin interaction parameter, respectively. $P(t)$ is a laser source with a Gaussian temporal profile. The term containing $K_{l}$ is a lattice thermal diffusion that is modeled to be proportional to the third power of the temperature difference ${ }^{32}$. In most cases, the thermal diffusion term could be neglected within a few tens of picoseconds in the case of an ultrafast demagnetization-remagnetization process of ferromagnetic metallic films ${ }^{9}, 33$. In addition, we also confirmed that a variation in the power of the thermal diffusion term does not modify the fitting result significantly, particularly in time scales less than $t<40 \mathrm{ps}$. The results of $3 \mathrm{TM}$ were applied to match the observed quantity of the normalized MOKE signal $\left(\left|\Delta \theta_{\text {Kerr }}\right| /\left|\Delta \theta_{\text {peak }}\right|\right)$, using $\Delta M \propto 1-\frac{\sqrt{1-\left(T_{S} / T_{C}\right)^{2}}-\sqrt{1-\left(T_{S}^{\text {peak }} / T_{C}\right)^{2}}}{\sqrt{1-\left(300 \mathrm{~K} / T_{C}\right)^{2}}-\sqrt{1-\left(T_{S}^{\text {peak }} / T_{C}\right)^{2}}}\left(\right.$ ref. $\left.{ }^{33}\right)$. A conventional 2-temperature model (2TM) was initially attempted, resulting in poor fittings, particularly under cycling external fields. A detailed comparison of fitting results by $3 \mathrm{TM}$ and $2 \mathrm{TM}$ is described in the Supplementary Note 6. A comparison between the experimental and $3 \mathrm{TM}$ results was carefully made, resulting in excellent agreement, as demonstrated in Supplementary Figs 8-10. Table 1 lists the fitting parameters.

The excellent fitting in the case of the external field angle $\theta_{\mathrm{H}}=23^{\circ}$ provides information on the temporal behaviors of $T_{\mathrm{s}}$, $T_{\mathrm{e}}$ and $T_{1}$, as plotted in Fig. 3 with the parameters listed in
Table 1 . In case of $n=15$, the equilibrium temperature, $T_{\text {eq }}$, where $T_{\mathrm{e}}$ becomes equal to $T_{1}$ for the first time is $\sim 937 \mathrm{~K}$ at $t=8.5 \mathrm{ps}$ under zero field. In the case of $n=5, T_{\mathrm{eq}} \sim 1232 \mathrm{~K}$ at $t=65$ ps under a zero field (not shown). As $T_{\mathrm{s}}$ is related to the magnetization, which is a magnetic ordering parameter, the lower $T_{\mathrm{s}}$ indicates a higher degree of magnetization (or equivalently larger remagnetization). The fitting results (Table 1) indicate that $G_{\mathrm{el}}$ increases in proportion to $n$, as previously reported for the case of 2TM under no external field ${ }^{34}$. The increased $G_{\text {el }}$ indicates an increase in the electron-lattice interaction, suggesting that the remagnetization converging to the equilibrium becomes faster. This interesting feature that the remagnetization becomes faster and larger with increasing $n$ possibly implies the controllability of the electron-lattice interaction with $n$.

The behaviors of $T_{\mathrm{s}}, T_{\mathrm{e}}$ and $T_{1}$ with the variation of the applied magnetic field strength were examined. In Fig. $3, T_{s}, T_{e}$ and $T_{1}$ under $1.70 \mathrm{kOe}$ was also plotted for $n=5$ and 15 . The equilibrium time $\left(t_{\mathrm{eq}}\right)$ required for $T_{\mathrm{s}}, T_{\mathrm{e}}$ and $T_{1}$ to reach $T_{\mathrm{eq}}$ was approximately several tens of picoseconds for $n=5$, whereas $t_{\text {eq }}$ is approximately a few picoseconds for $n=15$. The 3TM fitting indicated that $G_{\mathrm{el}}$ increases with $n$. With increasing $G_{\mathrm{el}}, t_{\mathrm{eq}}$ should be shortened. Therefore, $T_{\mathrm{s}}$ reaches $T_{\mathrm{eq}}$ more rapidly in the case of $n=15$ than for the case of $n=5$.

Attention should be drawn to another interesting point that in the case of $n=5$, under an applied field of $1.70 \mathrm{kOe}, t_{\mathrm{eq}}$ becomes significantly shorter, while there is little shortening for $t_{\text {eq }}$ in the case of $n=15$ as shown in Fig. 3. In other words, under a zero field, $T_{\mathrm{s}}$ follows $T_{\mathrm{e}}$ almost instantaneously on a demagnetization-remagnetization time scale, whereas under an external field, $T_{\mathrm{s}}$ follows $T_{\mathrm{e}}$ but with a delay: a clear separation of spin and electron temperatures was observed in the left part of Fig. 3b, c. As plotted in Fig. $3 \mathrm{c}$, the difference in $T_{\mathrm{s}}$ between $\mathbf{H}=0$ and $1.70 \mathrm{kOe}$ becomes largest $(204.8 \mathrm{~K})$ at $t=300 \mathrm{fs}$ and decreases thereafter in the case of $n=5$. Note that $T_{\mathrm{e}}$ is not changed significantly by $\mathbf{H}$ but only $T_{\mathrm{s}}$ is lowered substantially under an external magnetic field at the initial phase $(t<20 \mathrm{ps})$, clearly indicating that, there exists a MCE on the sub-picosecond timescale, as predicted in Fig. 1. The cooling of $204.8 \mathrm{~K}$, as shown in Fig. 3c, is enormous. Figure $4 \mathrm{a}$ shows the amount of cooling $\left(\Delta T_{\mathrm{s}}\right)$ under various $\mathbf{H}$ for $n=5$ and 15 samples.

Ultrafast magnetic cooling effect. The observed ultrafast giant MCE behavior is compared to a static MCE, which was analyzed from a field- and temperature-dependent magnetization behavior measured by means of superconducting quantum interference device. The specific heat of the samples was also measured by differential scanning calorimeter (DSC). The static MCE results are shown in the Supplementary Note 7. Total heat capacity values experimentally determined by DSC are $1.85 \times 10^{6} \mathrm{~J} \mathrm{~m}^{-3} \mathrm{~K}^{-1}(n=5)$ and $2.15 \times 10^{6} \mathrm{~J} \mathrm{~m}^{-3} \mathrm{~K}^{-1} \quad(n=15)$. 
The total heat capacity values measured by DSC are in a good agreement with the total sum of the heat capacities $\left(C_{1}+C_{\mathrm{e}}+C_{\mathrm{s}}\right)$
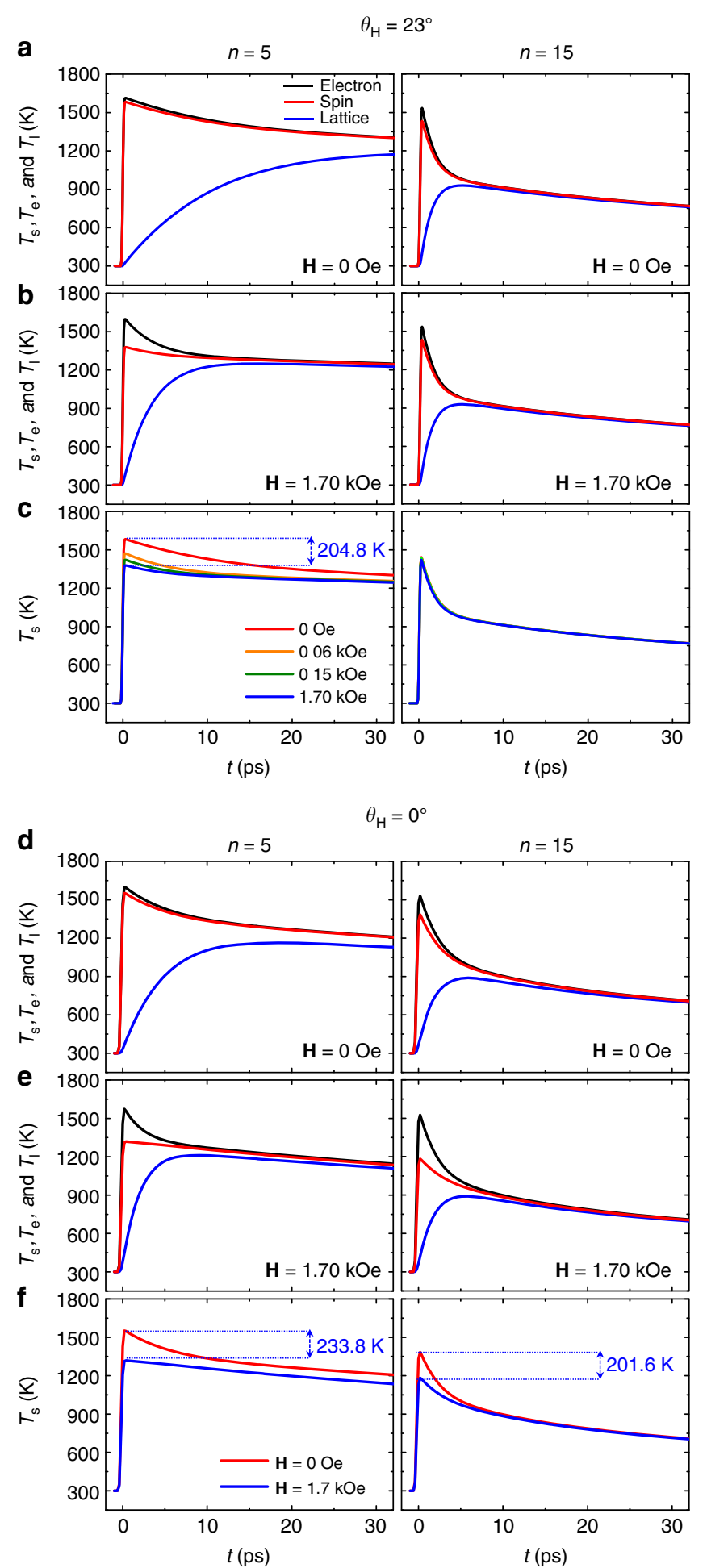

Fig. 3 Temperatures of electron, spin and lattice. Temporal behavior of $T_{\mathrm{s}}$ (red), $T_{\mathrm{e}}$ (black) and $T_{1}$ (blue) determined from 3TM analysis for $n=5$ (left) and 15 (right) with $\theta_{H}=23^{\circ} \mathbf{a}$ under a zero field and $\mathbf{b}$ external field of 1.70 kOe. $\mathbf{c} T_{\mathrm{s}}$ with respect to the delay for various fields of $\mathbf{H}$ between 0 and 1.70 kOe. $T_{\mathrm{s}}$ (red), $T_{\mathrm{e}}$ (black) and $T_{\mathrm{l}}$ (blue) determined from 3TM analysis for $n=5$ (left) and 15 (right) with $\theta_{\mathrm{H}}=0^{\circ}$ (d) under a zero field and (e) external field of $1.70 \mathrm{kOe}$. (f) $T_{\mathrm{s}}$ with respect to the delay for $\mathbf{H}=0$ and $1.70 \mathrm{kOe}$ from the $3 \mathrm{TM}$ fitting as in Table $1,1.63 \times 10^{6} \mathrm{~J} \mathrm{~m}^{-3} \mathrm{~K}^{-1}(n=5)$ and $3.19 \times 10^{6} \mathrm{~J} \mathrm{~m}^{-3} \mathrm{~K}^{-1}(n=15)$, respectively.

The cooling temperature $(\Delta T)$ in the conventional static MCE is given as follows:

$$
\Delta T=-\mu \int_{0}^{H_{\mathrm{Sat}}} \frac{T}{C}\left(\frac{\partial M}{\partial T}\right) \mathrm{d} H,
$$

where $\mu$ is permeability and $C$ is a total specific heat ${ }^{1}$. A huge cooling (large $\Delta T$ ) implies the drastic reduction of specific heat. In a static equilibrium case, a heat capacity with contributions from lattice, electron and spin will mostly increase if the system temperature increases, or at most, it should not decrease. The cooling temperature from the static MCE is found to be only 27 and $14 \mathrm{mK}$ for $n=5$ and 15 samples, substantially smaller than the cooling temperatures from the ultrafast MCE. Thus, the huge cooling observed in the present study cannot be explained within the context of the equilibrium MCE. We conclude that the observed giant MCE on the femtosecond timescale is fundamentally originated from the ultrafast non-equilibrium phenomenon. Unlike the equilibrium, spin, electron and lattice sub-system could be separated in a non-equilibrium and each sub-system independently behaves with separated value of specific heat on a femtosecond timescale. Thus, the temperature of the spin system, thermally separated from electron and lattice on ultrafast time scale, might be independently reduced with much smaller $C_{s}$ value: the replacement of $C$ with $C_{s}$ in Eq. (1) leads to a huge $\Delta T_{s}$.

Considering that the MCE is mainly a result of a forced alignment of spin, the observed ultrafast giant MCE should depend more sensitively on the strength of the aligning field. The aligning effective field is a vector sum of the external field $\left(\mathbf{H}_{\text {ext }}\right)$ and the anisotropy field $\left(\mathbf{H}_{\mathrm{k}}\right)$ of the sample. The measured $\mathbf{H}_{\mathrm{k}}$ was 12.6 and $6.6 \mathrm{kOe}$ for $n=5$ and 15 sample, respectively, as in the Supplementary Note 8 . We have repeated the same MCE experiment and the 3TM analysis with an external field direction normal to the film $\left(\theta_{\mathrm{H}}=0^{\circ}\right)$, where the effective field is expected to be stronger compared to the case of $\theta_{\mathrm{H}}=23^{\circ}$. The TR-MOKE signal is found to be fitted well again by the 3TM (see Supplementary Fig. 10), resulting in the temperature variation shown in Fig. 3d-f. Interestingly, the substantial MCE is now observable even for $n=15$ sample. The 3TM analysis implies that initially $(<5 \mathrm{ps})$ spin temperature is clearly separated and independently cooled by the $\mathbf{H}_{\text {ext }}$ on the ultrafast timescale before the temperatures reach equilibrium. Note that the time $\left(t_{\text {eq }}\right)$ required to reach equilibrium becomes shorter for the case of $n=15$ than for the case of $n=5$, as previously observed for the case of $\theta_{\mathrm{H}}=23^{\circ}$. It should be reminded that the resulting MCE is represented not by the value of $t_{\mathrm{eq}}$ itself but by the change of $t_{\mathrm{eq}}$ under external fields. $t_{\mathrm{eq}}$ is dramatically shortened again from 121 to 33.8 ps in case of $n=5$, while, to a lesser degree, from 25 to 15 ps in case of $n=15$. This is not so surprising since the ultrafast MCE due to the forced spin alignment under external fields should be rather a universal phenomenon.

We have carried out further experiments with the variation of pump fluence from 9.9 to $16.5 \mathrm{~mJ} \mathrm{~cm}^{-2}$. The experiments have been carried out with external fields applied normal to the films $\left(\theta_{\mathrm{H}}=0^{\circ}\right)$. The 3TM analysis of fluence-dependent TR-MOKE signals is described in Supplementary Fig. 10. The 3TM still fits to the data very well, where we used the same values for heat capacities of spin, electron and lattice as previous ones, while freeing the $G_{\mathrm{el}}, G_{\mathrm{es}}$ and $G_{\mathrm{ls}}$ parameters. For the case of $n=5$, field-cooling $(\Delta T)$, seems to be roughly saturated to $\sim 200$ $\mathrm{K}$ in all the pump fluences. It is interesting to note that $\Delta T$ increases with respect to the pump fluence for the case of $n=15$ (30 K for $9.9 \mathrm{~mJ} \mathrm{~cm}{ }^{-2}$ and $244 \mathrm{~K}$ for $16.5 \mathrm{~mJ} \mathrm{~cm} \mathrm{~cm}^{-2}$ fluence). With 

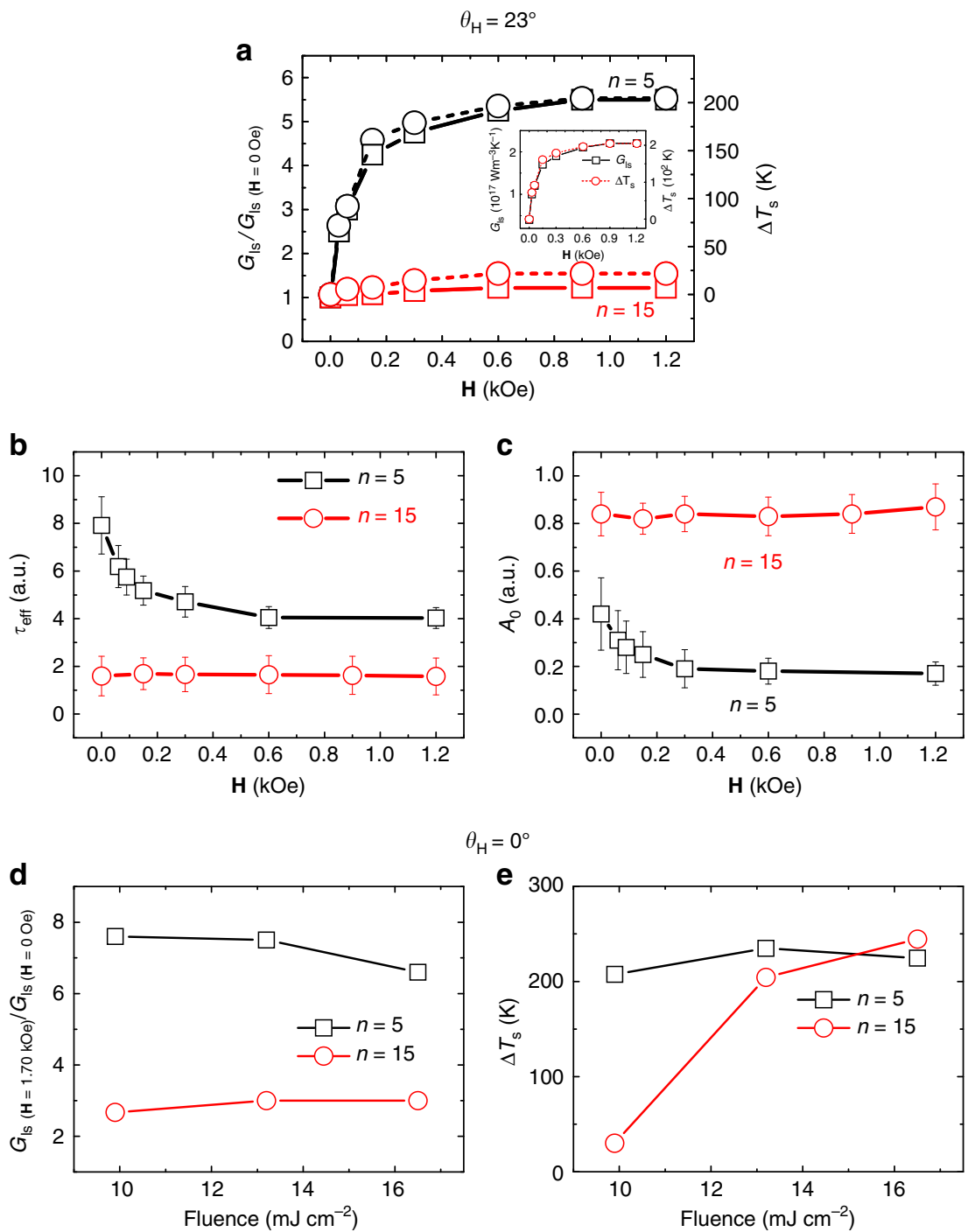

Fig. 4 Field- and fluence-dependent behavior. At $\theta_{H}=23^{\circ}$, a Field-dependent $G_{\mathrm{Is}} / G_{\mathrm{Is}(\mathbf{H}=0)}$ (open square solid line) and $\Delta T_{\mathrm{s}}$ (open circle dotted line) for $n=$ 5 (black) and 15 (red). Fitted values of $\tau_{\text {eff }} \mathbf{b}$ and $A_{O} \mathbf{c}$ under various fields for $n=5$ (open square) and 15 (open circle) samples. Fluence-dependency at $\theta_{H}$ $=0^{\circ}$ for $\mathbf{d} G_{\mathrm{Is}(\mathbf{H}=1.70 \mathrm{kOe})} / G_{\mathrm{Is}(\mathbf{H}=0)}$ and $\mathbf{e} \Delta T_{\mathrm{s}}$ for $n=5$ (open square) and 15 (open circle) samples

increasing the pump fluence and thus, providing much an excessive energy to the system, the existence of the MCE was observed even in the case of $n=15$, indicating that the ultrafast MCE on a femtosecond timescale could be a universal phenomenon.

In the context of the $3 \mathrm{TM}$, the shortened $t_{\mathrm{eq}}$ under an external field should be directly related to the increase of channel widths $\left(G_{\mathrm{el}}, G_{\mathrm{es}}\right.$ and $\left.G_{\mathrm{ls}}\right)$. One may consider that both $G_{\mathrm{ls}}$ and $G_{\text {es }}$ might be modified by an external field. A series of 3TM fittings to the data with $G_{\mathrm{ls}}$ and $G_{\mathrm{es}}$ being free parameters has been performed. Through fittings, we note that with increasing $\mathbf{H}$, the fitting value of $G_{\mathrm{es}}$ decreases, which might explain the experimental observation of $\Delta T_{s}$; On the other hand, decreased $G_{\text {es }}$ values cannot explain the trend of $t_{\text {eq }}$ with respect to $\mathbf{H}$, because a decreased $G_{\text {es }}$ would result in a longer $t_{\text {eq }}$, which is contradictory to the experimental observation. Therefore, only a possible option is that only $G_{\mathrm{ls}}$ changes with $\mathbf{H}$. In Fig. $4 \mathrm{a}$, the field-dependent $G_{\mathrm{ls}}$ normalized by $G_{\mathrm{ls}}$ at $\mathbf{H}=0 \quad\left(G_{\mathrm{ls}} / G_{\mathrm{ls}(\mathbf{H}=0)}\right)$ together with $\Delta T_{\mathrm{s}}$ for $n=5$ and 15 sample is plotted. In the case of $n=15$, relatively small modification of $G_{\mathrm{ls}}$ was observed for $\theta_{\mathrm{H}}=23^{\circ} . G_{\mathrm{ls}} / G_{\mathrm{s}(\mathbf{H}=0)} \sim 1.2$ at $\mathbf{H}=1.70 \mathrm{kOe}$ as shown in Fig. 4a.
On the other hand, in the case of $n=5, G_{\mathrm{ls}}$ is $\sim 4 \times 10^{16} \mathrm{~W} \mathrm{~m}^{-3} \mathrm{~K}$ at $\mathbf{H}=0 \mathrm{Oe}$ and increases substantially to be saturated at $2.2 \times 10^{17} \mathrm{~W} \mathrm{~m}^{-3} \mathrm{~K}$ for $\mathbf{H}=0.90 \mathrm{kOe}$, above which $G_{\mathrm{ls}}$ remains unchanged, as shown in the inset of the figure. $G_{\mathrm{ls}} / G_{\mathrm{ls}(\mathbf{H}=0)} \sim 5.5$ at $\mathbf{H}=1.70 \mathrm{kOe}$. Note that the saturation field of $G_{\mathrm{ls}}$ is similar to the saturation field of the loop in Fig. 2b. Similarly, for $n=10$, $G_{\mathrm{ls}}$ is $\sim 1.2 \times 10^{17} \mathrm{~W} \mathrm{~m}^{-3} \mathrm{~K}$ at $\mathbf{H}=0 \mathrm{Oe}$ and increases to a saturation of $6.0 \times 10^{17} \mathrm{~W} \mathrm{~m}^{-3} \mathrm{~K}$ at $\mathbf{H}=0.60 \mathrm{kOe}$ similar to saturation field of the hysteresis loop in Fig. $2 \mathrm{~b}$, leading to $G_{\mathrm{ls}} / G_{\mathrm{ls}(\mathbf{H}=0)} \sim 5.0$. Note the striking similarity in the trend between $G_{\mathrm{ls}} / G_{\mathrm{ls}(\mathbf{H}=0)}$ and $\Delta T_{\mathrm{s}}$, suggesting that the field-cooling of spin temperature $\left(\Delta T_{\mathrm{s}}\right)$ is involved directly in the modification of $G_{l s}$ under an external magnetic field. The modification of $G_{l s}$ by the external field is phenomenologically linked to the ultrafast MCE, which is expected from the fact that the extra energy pumped by the laser pulse should be dissipated eventually into the lattice, while the spin temperature is affected by the external magnetic field. The effective modification of energy paths $\left(G_{\mathrm{el}}, G_{\mathrm{es}}\right.$ and $G_{\mathrm{ls}}$ ) is not new. In the case of semiconductor, where the 2TM model (electron and lattice temperatures) has been effectively applied, the modification of $G_{\mathrm{el}}$ by pump fluence has been 
reported ${ }^{35-37}$. The field-dependent lattice-spin and electron-spin interaction have recently been reported for molecules composed of transition metals ${ }^{38,39}$. The modified $G_{\mathrm{ls}}$ should modify the temperatures of the spin and lattice. If $G_{\mathrm{ls}}$ becomes larger under an external field, the lattice will absorb the heat in the spin system more rapidly, leading to a larger MCE.

The remagnetization curve after the maximal demagnetization was fitted for $t<10 \mathrm{ps}$ with an exponential function. The exponential fitting with $A_{0} \mathrm{e}^{-\frac{\tau_{\text {eff }}}{4}}$ for the initial remagnetization is used widely ${ }^{40,41}$, where $A_{0}$ is approximately proportional to $\left(T_{\mathrm{s}}^{\max }-T_{\text {eq }}\right)$ and $\tau_{\text {eff }}$ is an effective relaxation time. In particular, it was reported that $\tau_{\text {eff }}$ may be a direct measure of an effective characteristic time of the lattice-spin interaction ${ }^{40-43}$. As discussed above, $t_{\mathrm{eq}}$ is shortened significantly in the case of $n=5$. A smaller $t_{\mathrm{eq}}$ due to the ultrafast magnetic cooling under the field will result in a smaller $\tau_{\text {eff }}$ as well. Indeed, in the case of $n=5, \tau_{\text {eff }}$ decreases with increasing $\mathbf{H}$, as shown in Fig. $4 \mathrm{~b}$, consistently confirming the MCE as analyzed based on 3TM.

The 3TM calculation suggests that, although $t_{\text {eq }}$ becomes shorter under $\mathbf{H}, T_{\mathrm{eq}}$ remains the same, irrespective of $\mathbf{H}$. Therefore, it is expected that $A_{0}$ should become smaller with increasing magnetic field, because $T_{\mathrm{s}}^{\max }$ is cooled under the field. A decrease in $A_{0}$ with respect to the field is observed for $n=5$ as shown in Fig. 4c. For $n=15$, no significant change in $G_{\mathrm{ls}}$ and thus, no substantial MCE is observed according to the 3TM analysis, suggesting that $A_{0}$ is constant, as confirmed in Fig. 4c. In the case of $n=5$, both $A_{0}$ and $\tau_{\text {eff }}$ at a $\mathbf{H}=0$ field reduces to almost half at a field higher than $0.60 \mathrm{kOe}$. Considering that $A_{0}$ is related to the degree of spin temperature cooling and $\tau_{\text {eff }}$ is also related to $t_{\mathrm{eq}}$, the contributions from both parameters should be included in $G_{\mathrm{ls}}$.

The field dependence of $\tau_{\text {eff }}$ has already been observed experimentally for a semiconductor system ${ }^{44}$. In the semiconductor, the contribution from $T_{\mathrm{e}}$ is negligible and $\tau_{\mathrm{eff}}$ is approximated to be the characteristic relaxation time $\left(\tau_{1 \mathrm{~s}}\right)$ of the lattice-spin interaction channel, where $\tau_{\mathrm{ls}} \sim C_{\mathrm{s}} / G_{\mathrm{ls}}{ }^{42}, 43$. It was reported for such a system that the $C_{\mathrm{s}}$ value is proportional to the external magnetic field ${ }^{45}, 46$. Therefore, the decrease in $\tau_{l s}$ under the field was attributed to the increased $G_{\mathrm{Is}}$.

It should be mentioned that the ratio $G_{\mathrm{ls}} / G_{\mathrm{ls}(\mathbf{H}=0)}$ is believed to represent the variation of the channel width of energy transfer under external magnetic field between the spin and the lattice. The dissipated heat from spin should be better transferred to lattice with a larger channel width under external magnetic fields. We have carried out experiment for different pump fluences of 9.9 to $16.5 \mathrm{~mJ} \mathrm{~cm}^{-2}$ and analyzed with $3 \mathrm{TM}$ model. Interestingly, although $G_{\mathrm{ls}}$ as well as $G_{\mathrm{es}}$ and $G_{\mathrm{el}}$ is effectively reduced with respect to the fluence as in Table 1 , there seems to be no significant correlation between the ratio $G_{\mathrm{ls}} / G_{\mathrm{s}(\mathbf{H}=0)}$ and the fluence as in Fig. $4 \mathrm{~d}$, implying that the ratio could be a practically good parameter reflecting the effective channel width variation by the magnetic field, irrespective of the fluence variation. If the direct fundamental channel between the spin and the lattice is only related to the spin-orbit coupling, field-dependent varying quantities such as magnetostriction related to the spin-orbit coupling might play a role in modifying the $G_{\mathrm{ls}}{ }^{47}, 48$. We encourage further theoretical works regarding the variation of the energy transfer channel width under an external magnetic field.

As seen in Fig. 4e, with proper tuning of external parameters such as the pump fluence and the external field direction, the MCE was found to be observable. The giant ultrafast MCE by cooling the spin temperature up to $200 \mathrm{~K}$ is clearly manifested at the fluence of $16.5 \mathrm{~mJ} \mathrm{~cm}^{-2}$, implying that the MCE by the forced spin alignment could be universal phenomenon even on a sub-ps timescale. The observed ultrafast giant MCE opens a new possibility in engineering the mechanism of heating and energy transfer on a femtosecond timescale. Controlled MCE behavior by an external magnetic field, combined with the easy tunability of $G_{\mathrm{Is}}$ and other magnetic properties for the nanostructured $\mathrm{Co} / \mathrm{Pt}$ multilayer, will be particularly useful and promising for spintronic applications with devices operating at an ultrafast speed, where the management of heat and energy transfer becomes increasingly important.

In summary, the dynamics of time-resolved hysteresis loop in Co/Pt multilayers were investigated systematically with a repeat number $\mathrm{n}$ of 5, 10 and 15. Pump-beam modulated TR-MOKE measurements were analyzed comprehensively using 3TM. From an experimental demonstration of the existence of giant MCE on an ultrafast time scale, it was found that MCE can be controlled via effective manipulation of the lattice-spin interaction through modification of multilayer parameter and pump fluence under an external field. This study is not only a proof-of-principle experiment but might provide the possibility of future applications utilizing the femtosecond and/or picoseconds magnetic cooling phenomenon, opening a door for studies and applications of ultrafast magnetic cooling on a sub-picosecond timescale.

\section{Methods}

MOKE measurement. Time-resolved magneto-optical Kerr effect (TR-MOKE) measurements in a pump-probe geometry (Supplementary Fig. 1) were carried out for $\mathrm{Co} / \mathrm{Pt}$ multilayers. Mechanical chopping modulation at $750 \mathrm{~Hz}$ was implemented in a pump-beam path, combined with a lock-in amplifier to discriminate purely pump-induced magnetization changes to improve the signal-to-noise ratio. The measurement at $44 \mathrm{~Hz}$ modulating in synchronization of pump-probe stroboscopic frequency with the field-cycling frequency was also carried out to confirm the result at $750 \mathrm{~Hz}$ modulation. TR-MOKE signal with the probe-beam modulation was also taken. The pump pulses were generated by a Ti:sapphire multipass amplifier operating at $3 \mathrm{kHz}$ repetition rate with a center wavelength of $780 \mathrm{~nm}$ and a pulse duration of $25 \mathrm{fs}$. The probe pulses with the same wavelength were generated by a beam splitter. The Wollaston polarizer was positioned to split out the s- and the p-polarization component in front of the two photodiodes, each of which detects the s- and p-polarization component, respectively. This measurement leads to a difference between the s- and p-polarization components of the probe pulses modified by MOKE at the reflection off a film surface. The pump fluence was varied from 9.9 to $16.5 \mathrm{~mJ} \mathrm{~cm}^{-2}$ and the probe fluence was 0.3

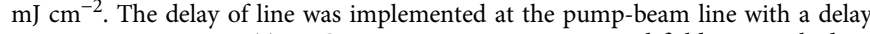
time up to 700 ps. For TR-MOKE measurement, an external field was applied with an angle of 0 and $23^{\circ}$ to the surface normal of the sample, and swept up to $1.70 \mathrm{kOe}$, to measure the MOKE hysteresis loops at each delay. The external magnetic field was cycled 15 times to produce the averaged TR-MOKE hysteresis loop at each delay. The time step of each delay was $50 \mathrm{fs}$.

Samples. $[\operatorname{Co}(6.2 \AA) / \operatorname{Pt}(7.7 \AA)]_{n}$ multilayer films with $n=5,10$ and 15 were deposited by dc magnetron sputtering on Si substrates, and capped by a $22 \AA \mathrm{Pt}$ to protect the oxidation of the surface. The structure of the $\mathrm{Co} / \mathrm{Pt}$ multilayers with well-defined interfaces was confirmed by a low angle X-ray diffraction and the extended X-ray absorption fine structure analysis. All the films exhibited PMA and typical saturation magnetization values ${ }^{19-23}$, as confirmed by a vibrating sample magnetometer (VSM).

Data availability. The data that support the findings of this study are available from the corresponding authors upon reasonable request.

Received: 19 July 2015 Accepted: 28 July 2017

Published online: 06 October 2017

\section{References}

1. Phan, M.-H. \& Yu, S.-C. Review of the magnetocaloric effect in manganite materials. J. Magn. Magn. Mater. 308, 325-340 (2007).

2. Pecharsky, V. K. \& Gschneidner, K. A. Jr. Giant magnetocaloric effect in $\mathrm{Gd}_{5}\left(\mathrm{Si}_{2} \mathrm{Ge}_{2}\right)$. Phys. Rev. Lett. 78, 4494-4497 (1997).

3. Tegus, O., Brück, E., Buschow, K. H. J. \& Boer, F. R. Transition-metal-based magnetic refrigerants for room-temperature applications. Nature 415, 150-152 (2002).

4. Provenzano, V., Shapiro, A. J. \& Shull, R. D. Reduction of hysteresis losses in the magnetic refrigerant $\mathrm{Gd}_{5} \mathrm{Ge}_{2} \mathrm{Si}_{2}$ by the addition of iron. Nature 429, 853-857 (2004). 
5. Campos, A. et al. Ambient pressure colossal magnetocaloric effect tuned by composition in $\mathrm{Mn}_{1-\mathrm{x}} \mathrm{Fe}_{\mathrm{x}} \mathrm{As}$. Nat. Mater. 5, 802-804 (2006).

6. Liu, J., Gottschall, T., Skokov, K. P., Moore, J. D. \& Gutfleisch, O. Giant magnetocaloric effect driven by structural transitions. Nat. Mater. 11, 620-626 (2012).

7. Moya, X. et al. Giant and reversible extrinsic magnetocaloric effects in $\mathrm{La}_{0.7} \mathrm{Ca}_{0.3} \mathrm{MnO}_{3}$ films due to strain. Nat. Mater. 12, 52-58 (2012).

8. Zhu, H. et al. Magnetocaloric effects in a freestanding and flexible graphenebased superlattice synthesized with a spatially confined reaction. Nat. Commun. 5, 3960 (2014).

9. Beaurepaire, E., Merle, J.-C., Daunois, A. \& Bigot, J.-Y. Ultrafast spin dynamics in ferromagnetic Nickel. Phys. Rev. Lett. 76, 4250-4253 (1996).

10. Radu, I. et al. Transient ferromagnetic-like state mediating ultrafast reversal of antiferromagnetically coupled spins. Nature 472, 205-209 (2011).

11. Stanciu, C. D. et al. Ultrafast interaction of the angular momentum of photons with spins in the metallic amorphous alloy GdFeCo. Phys. Rev. Lett. 98, 207401 (2007).

12. Mendil, J. et al. Resolving the role of femtosecond heated electrons in ultrafast spin dynamics. Sci. Rep. 4, 3980 (2014).

13. Koopmans, B., Ruigrok, J. J. M., Dalla Longa, F. \& Jonge, W. J. M. Unifying ultrafast magnetization dynamics. Phys. Rev. Lett. 95, 267207 (2005).

14. Koopmans, B. et al. Explaining the paradoxical diversity of ultrafast laserinduced demagnetization. Nat. Mater. 9, 259-265 (2010).

15. Atxitia, U. et al. Micromagnetic modeling of laser-induced magnetization dynamics using the Landau-Lifshitz-Bloch equation. Appl. Phys. Lett. 91, 232507 (2007)

16. Atxitia, U., Chubykalo-Fesenko, O., Walowski, J., Mann, A. \& Münzenberg, M. Evidence for thermal mechanisms in laser-induced femtosecond spin dynamics. Phys. Rev. B 81, 174401 (2010).

17. Atxitia, U. \& Chubykalo-Fesenko, O. Ultrafast magnetization dynamics rates within the Landau-Lifshitz-Bloch model. Phys. Rev. B 84, 144414 (2010).

18. Müller Georg, M. et al. Spin polarization in half-metals probed by femtosecond spin excitation. Nat. Mater. 8, 56-61 (2008).

19. Hashimoto, S., Ochiai, Y. \& Aso, K. Ultrathin $\mathrm{Co} / \mathrm{Pt}$ and $\mathrm{Co} / \mathrm{Pd}$ multilayered films as magneto-optical recording materials. J. Appl. Phys. 67, 2136-2142 (1990).

20. Hashimoto, S., Ochiai, Y. \& Aso, K. Fim thickness dependence of magnetooptical and magnetic properties in $\mathrm{Co} / \mathrm{Pt}$ and $\mathrm{Co} / \mathrm{Pd}$ multilayers. J. Appl. Phys. 67, 4429-4431 (1990).

21. Zeper, W. B., Kesteren, H. W., Jacobs, B. A. J., Spruit, J. H. M. \& Carcia, P. F. Hysteresis, microstructure, and magneto-optical recording in $\mathrm{Co} / \mathrm{Pt}$ and $\mathrm{Co} / \mathrm{Pd}$ multilayers. J. Appl. Phys. 70, 2264-2271 (1991).

22. Yakushiji, K. et al. Ultrathin $\mathrm{Co} / \mathrm{Pt}$ and $\mathrm{Co} / \mathrm{Pd}$ superlattice films for $\mathrm{MgO}$-based perpendicular magnetic tunnel junctions. Appl. Phys. Lett. 97, 232508 (2010).

23. Zeper, W. B., Greidanus, F. J. A. M., Carcia, P. F. \& Fincher, C. R. Perpendicular magnetic anisotropy and magnetooptical Kerr effect of vapor deposited Co/Ptlayered structures. J. Appl. Phys. 65, 4971-4975 (1989).

24. Cheskis, D., Porat, A., Szapiro, L., Potashnik, O. \& Bar-Ad, S. Saturation of the ultrafast laser-induced demagnetization in nickel. Phys. Rev. B 72, 014437 (2005).

25. Liu, X. D. et al. Single laser pulse induced dynamic magnetization reversal mechanism of perpendicularly magnetized $\mathrm{L}_{0}$ FePt films. J. Appl. Phys. 106, 053907 (2009).

26. Bunce, C. et al. Laser-induced magnetization switching in films with perpendicular anisotropy: a comparison between measurements and a multimacrospin model. Phys. Rev. B 81, 174428 (2010).

27. Luo, J. et al. Dynamics of photo-enhanced magneto-crystalline anisotropy in diluted ferromagnetic GaMnAs. Solid State Commun. 150, 1419-1421 (2010).

28. Wilks, R., Hughes, N. D. \& Hicken, R. J. Investigation of ultrafast spin dynamics in a Ni thin film. J. Appl. Phys. 91, 8670-8672 (2002).

29. Guidoni, L., Beaurepaire, E. \& Bigot, J.-Y. Magneto-optics in the ultrafast regime: thermalization of spin populations in ferromagnetic Films. Phys. Rev. Lett. 89, 017401 (2002).

30. Koopmans, B., Kampen, M., Kohlhepp, J. T. \& Jonge, W. J. M. Ultrafast magneto-optics in nickel: magnetism or optics? Phys. Rev. Lett. 85, 844-847 (2000).

31. Vomir, M., Andrade, L. H. F., Beaurepaire, E., Albrecht, M. \& Bigot, J.-Y. Ultrafast magnetization dynamics investigated in real space (invited). J. Appl. Phys. 99, 08A501 (2006)

32. Lu, G., Wang, X. D., Duan, Y. Y. \& Li, X. W. Effects of non-ideal structures and high temperatures on the insulation properties of aerogel-based composite materials. J. Non-Cryst. Solid 357, 3822-3829 (2011).

33. Bigot, J.-Y., Vomir, M., Andrade, L. H. F. \& Beaurepaire, E. Ultrafast magnetization dynamics in ferromagnetic cobalt: the role of the anisotropy. Chem. Phys. 318, 137-146 (2005).

34. Kim, C. H. et al. Coherent phonon control via electron-lattice interaction in ferromagnetic Co/Pt multilayers. Sci. Rep. 6, 22054 (2016).
35. Fujimoto, J. G., Liu, J. M., Ippen, E. P. \& Bloembergen, N. Femtosecond laser interaction with metallic tungsten and nonequilibrium electron and lattice temperatures. Phys. Rev. Lett. 53, 1837-1840 (1984).

36. Lin, J., Zhigilei, L. V. \& Celli, V. Electron-phonon coupling and electron heat capacity of metals under conditions of strong electron-phonon nonequilibrium. Phys. Rev. B 77, 075133 (2008).

37. Qiu, T. Q. \& Tien, C. L. Short-pulse laser heating on metals. Int. J. Heat Mass Transfer 35, 719-726 (1992).

38. Gilch, P., Pöllinger-Dammer, F., Musewald, C., Michel-Beyerle, M. E. \& Steiner, U. E. Magnetic field effect on picosecond electron transfer. Science 281, 982-984 (1998).

39. Feilitzsch, T. et al. Magnetic field and temperature dependencies shed light on the recombination kinetics of a transition metal donor/acceptor system. J. Am. Chem. Soc. 127, 15228-15235 (2005).

40. Weber, M. C., Hillebrands, B., Moshnyaga, V. \& Samwer, K. Spin-lattice relaxation phenomena in manganite $\mathrm{La}_{0.7} \mathrm{Sr}_{0.3} \mathrm{MnO}_{3}$ thin films. Europhys. Lett. 73, 285-291 (2006).

41. Laraoui, A. et al. Study of individual ferromagnetic disks with femtosecond optical pulses. J. Appl. Phys. 101, 09C105 (2007).

42. Lobad Ahmed, I., Averitt Richard, D., Kwon, C. \& Taylor, A. J. Spin-lattice interaction in colossal magnetoresistance manganites. Appl. Phys. Lett. 77, 4025-4027 (2000).

43. Taylora, A. J. et al. Femtosecond studies of mixed valence state formation in strongly correlated electron materials. Phys. B Cond. Matter 312, 640-646 (2002).

44. Strutz, T., Witowski, A. M. \& Wyder, P. Spin-lattice relaxation at high magnetic fields. Phys. Rev. Lett. 68, 3912-3915 (1992).

45. Flokstra, J., Gerritsma, G. J. \& Marel, L. C. Spin-lattice relaxation in potassium chromium alum. Phys. B Cond. Matter 94, 53-59 (1978).

46. Reeves, M. E. et al. Field-dependent specific heat of polycrystalline $\mathrm{YBa}_{2} \mathrm{Cu}_{3} \mathrm{O}_{7-\mathrm{x}}$. Phys. Rev. B 40, 4573-4584 (1989).

47. Ashworth, H., Sengupta, D., Schnakenberg, G., Shapiro, L. \& Berger, L. Galvanomagnetic effects, magnetostriction, and spin-orbit interaction in $\mathrm{Cu}-$ $\mathrm{Ni}-\mathrm{Fe}$ and other ferromagnetic nickel alloys. Phys. Rev. 185, 792-797 (1969).

48. Ruqian, W. \& Freeman, A. J. Spin-orbit induced magnetic phenomena in bulk metals and their surfaces and interfaces. J. Magn. Magn. Mater. 200, 498-514 (1999).

\section{Acknowledgements}

This study was supported by Korea Research Foundation (NRF) grant No. 2015R1D1A3A01020686 and a KBSI Grant D37614. This research has been supported in part by Global Research Laboratory Program [Grant No 2009-00439] and by Max Planck POSTECH/KOREA Research Initiative Program [Grant No 2016K1A4A4A01922028] through the National Research Foundation of Korea (NRF) funded by Ministry of Science, ICT \& Future Planning

\section{Author contributions}

J.-H.S., A.A.S, C.-H.K and S.-Y.P. collected data and performed all the analyses. K.M.L. and J.-R.J. fabricated all the samples. D.-H.K. and D.E.K. are involved in the design of experiments. All authors discussed the results and commented on the manuscript.

\section{Additional information}

Supplementary Information accompanies this paper at doi:10.1038/s41467-017-00816-w.

Competing interests: The authors declare no competing financial interests.

Reprints and permission information is available online at http://npg.nature.com/ reprintsandpermissions/

Publisher's note: Springer Nature remains neutral with regard to jurisdictional claims in published maps and institutional affiliations.

Open Access This article is licensed under a Creative Commons Attribution 4.0 International License, which permits use, sharing adaptation, distribution and reproduction in any medium or format, as long as you give appropriate credit to the original author(s) and the source, provide a link to the Creative Commons license, and indicate if changes were made. The images or other third party material in this article are included in the article's Creative Commons license, unless indicated otherwise in a credit line to the material. If material is not included in the article's Creative Commons license and your intended use is not permitted by statutory regulation or exceeds the permitted use, you will need to obtain permission directly from the copyright holder. To view a copy of this license, visit http://creativecommons.org/ licenses/by/4.0/.

(C) The Author(s) 2017 